This is an authors' version of the article published in "Neurocomputing". The final authenticated version is available online at: https://doi.org/10.1016/j.neucom.2020.03.116

\title{
A neural blockchain for a tokenizable e-Participation model
}

\author{
Francisco Luis Benítez-Martínez*ab1, María Visitación Hurtado-Torres ${ }^{\mathrm{a}}$ Esteban Romero-Frías $^{\mathrm{b}}$ \\ ${ }^{a}$ Department of Languages and Computer Systems, University of Granada, ETSIIT, Periodista Daniel Saucedo Aranda s/n, 18071, Granada, \\ Spain

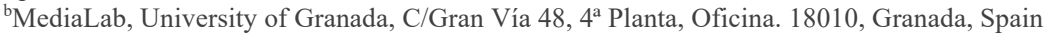

\section{Highlights}

- A tokenizable e-Participation model will develop new local engagement.

- Using a "democratic token" will help governments to overcome the governance deficit.

- Building a BaaS neural network within a cloud framework is a scalable and sustainable solution.

- A Neural Distributed Ledger (NDL) is a step beyond developing a 3D blockchain system.

- A dApp called VoteKeeper uses Smart Contracts as a block producer in a virtual token.

\begin{abstract}
Currently, Distributed Ledger Technologies (DLTs) and, especially, Blockchain technology represent a great opportunity for public institutions to improve citizen participation and foster democratic innovation. These technologies facilitate the simplification of processes and provide secure management of recorded data, guaranteeing the transmission and public transparency of information. Based on the combination of a Blockchain as a Service (BaaS) platform and G-Cloud solutions, our proposal consists of the design of an e-Participation model that uses a tokenizable system of the actions and processes undertaken by citizens in participatory processes providing incentives to promote greater participation in public affairs. In order to develop a sustainable, scalable and resilient e-Participation system, a new blockchain concept, which organizes the blocks as a neural system, is combined with the implementation of a virtual token to reward participants. Furthermore, this virtual token is deployed through a smart contract that the block itself produces, containing information about the transaction and all the documents involved in the process. Finally, our Neural Distributed Ledger (NDL) framework facilitates the interconnection of blockchain networks in a transparent, certified, secure, auditable, scalable and traceable way.
\end{abstract}

Keywords: Governance, neural blockchain, e-Participation, tokenization, smart citizen, open government

Reference: Benítez-Martínez, F.L., Hurtado-Torres, M. V., and Romero-Frías, E. (2020). A
neural blockchain for a tokenizable e-Participation model. Neurocomputing,
https://doi.org/10.1016/j.neucom.2020.03.116

\footnotetext{
$1 *$ Corresponding author.

Email addresses: flbenitez@,correo.ugr.es (F.L. Benítez-Martínez), mhurtado@ugr.es (M.V. Hurtado-Torres), erf@ugr.es (E. Romero-Frías).
} 
This is an authors' version of the article published in "Neurocomputing". The final authenticated version is available online at: https://doi.org/10.1016/j.neucom.2020.03.116

\section{$1 \quad$ Introduction}

In 2009, Satoshi Nakamoto [1] described how to use Blockchain technology as a cryptographic management system in a single, distributed registry. This seminal contribution was a milestone in the quest to make a digital ecosystem secure and verifiable - conditions that had not been prioritized in the original design of the Internet and the Web. Thus, blockchain technology opened up a new era of trust in our social, economic and political transactions, disrupting and transforming the value chain in many different areas.

The financial sector was the most dynamic in adopting the changes incorporated by the application of Blockchain technology. Nakamoto himself designed Bitcoin as its first application and this was quickly followed by a whole new wave of cryptocurrencies based in the same distributed architecture. The Fintech sector has developed a set of dApps (mobile applications using blockchain technology that can be public, permissioned or federated) that permit multiscreen integration in a distributed manner. Furthermore, the Ethereum platforms have deployed the use of smart contracts in several ways [2].

Simultaneously, newly designed types of DLTs have fostered the development of new formats and opportunities due to more advanced node topologies such as Hedera Hashgraph [3] or Tangle, from the IOTA Foundation [4]. Both of these belong to a new generation of DLTs that are completely different from the first blockchains.

First and second generation DLTs are oriented to establish tokens with added economic value, either via a mining system or through the value they obtain for the transactions they register in each of the blocks they build. Additionally, their management is based on the ability to generate an economic reward and-depending on its work scheme (public, private or consortium) - their transaction verification systems and consensus mechanisms make the DLTs difficult to scale. Also, the size of the network's validating nodes makes this complex.

Our proposal does not use traditional consensus systems: the blocks are created from the smart contracts themselves and the transactions involve no economic expenditure. Since it is a BaaS system based on a 3D blockchain network which simulates a neural system, the management, validation and production of the blocks is totally different from current existing systems.

Developing new DLTs is an opportunity to manage complex processes that simplify governance practices in many different sectors beyond that of finance. As long as DLTs can guarantee data structure and security, they represent a promising opportunity for the management and assurance of transparency and efficiency in public sector transactions while maintaining information security. This is why Blockchain application in the public sector has received increasing attention from government, practitioners and consulting services [5].

Another important feature is their temporary sealing capacity and the inalterability of the data they contain, as well as the reduced risk of unauthorized access and data manipulation-even by insiders who previously had access to documents and could potentially alter their contents. In the public sector, blockchain also allows the configuration of effective management systems [6] to achieve better accountability through standard, scalable e-Government models at different administrative levels. Countries like Estonia or Sweden are testing solutions of this type; other countries have created governmental offices to lead digital transformation and deploy, among others, blockchain projects, as is the case of Australia's Digital Transformation Agency, the U.S. Digital Service, or the UK Government's Digital Service.

Citizen participation has recently become one of the most important topics in public government. Welch [7] defined e-Participation as a part of e-Government that especially focuses on citizen engagement in deliberation and/or decision-making in public affairs. Although the topic is not new-early practice and theory were reported in the United States in the 1960s and 1970s [8][9] — current demands for a more participatory democracy in a context in which digital tools facilitate new means of interaction have brought participation to the forefront. Citizen engagement in participatory processes is a cornerstone for strengthening and supporting a sound modern democracy [10].

Distributed Ledger Technologies can provide solutions in the development of a new democratic management framework to empower citizens to participate in public issues in ways that go beyond voting or administrative tasks. The digital transformation of the administration to foster citizen empowerment is a governmental duty and a civic demand that nowadays is more feasible than ever before. This is due to the disruption brought about by DLTs [11] and the cloud solutions that facilitate a platform approach to the design of new public management tools, including participatory ones [12]. Authors such as Huang and Karduck [13] have proposed methodologies to implement this type of system but there are still significant opportunities to design and experiment with new solutions to extend democratic participation in public governance.

Therefore, blockchain technologies in platform models [14] represent a unique opportunity to manage new enriched participatory processes organized by public institutions in a more effective and efficient manner [15]. Thus, the objective of this paper is to propose an e-Participation model that uses DLTs systems [16] [17] to implement a new 
This is an authors' version of the article published in "Neurocomputing". The final authenticated version is available online at: https://doi.org/10.1016/j.neucom.2020.03.116

tool to facilitate and empower citizen participation. The model integrates the opportunities offered by DLTs systems [18] in relation to public governance processes in order to develop new tools for citizen empowerment. To this end, we will describe a "tokenizable" participation system that helps local government to improve participatory processes using electronic voting tools based on a neuronal blockchain network that facilitates improved collection of citizens' preferences in the daily management of cities. The proposed system is based on a new use of smart contracts [19] that replace a block production approach and the need for miners to generate network transactions [20]. Our system offers a neural network of connected blockchains to use smart contracts as transaction managers through participating nodes. In addition, this model permits the development of more sustainable and eco-friendly blockchains [21].

We propose the use of a G-Cloud system to allow optimal deployment with a highly scalable, reproducible capacity in any environment, following a proven methodology, as outlined in the White Paper on Cloud Computing for eGovernance [22]. Consequently, this paper proposes the application of new processes and procedures with the use of blockchains as a platform within a G-Cloud based framework, facilitating citizenship engagement in local eParticipation projects.

The structure of our paper is as follows. The next section presents a literature review of successful platforms used in the study in order to highlight the novelty of our proposal. The third section describes our model: a tokenizable eParticipation model based on a platform design [23]. The fourth section details the structure and theoretical system of RETIS, a neural blockchain network prepared to be launched in a cloud environment under a BaaS (Blockchain as a Service) approach [24]. In the final section the paper discusses the opportunities offered by this new e-Participation framework using blockchain as a platform and offers directions for future work.

\section{2 e-Participation systems: Technological Background and related works}

An e-Participation system is considered as one of any e-Government's core components (United Nations, 2014), consisting of the use of technology to mediate and facilitate dialog between citizens, politicians and administration [25] in order to enable effective, concurrent public participation and feedback, as well as to experiment with new methods of innovative democratic participation [26][27]. Macintosh [10] defined e-Participation as "the use of information and communication technologies to broaden and deepen political participation by enabling citizens to connect with one another and with their elected representatives" and, according to the UNDESA report [28], "eParticipation is recognized as a political action that is facilitated by digital technologies in order to revive citizens' sovereignty, through participation in the processes of policy and political decision-making".

The United Nations e-Government Survey 2014 report [29] describes a three-level e-Participation model: eInformation, e-Consultation and e-Decision-making. There is a progressive move from a more "passive" role linked to information access and transparency to a more "active" one based on deliberation, decision-making and co-creation [30]. Similarly, Macintosh [10] also pointed to three levels of e-Participation: e-Enabling, e-Engaging and eEmpowering, going from a more passive role for citizens to active involvement that to some extent returns sovereignty to citizens. Several various authors [31] [32] [33] have suggested that citizen empowerment is one of the key ingredients for successful citizen participation over time in consultation and decision-making processes.

Digital platforms have become an essential instrument in facilitating participation. Also known as crowd-civic systems [34], they can be defined as "socio-technical systems blending people, digital technologies, and data for civic engagement purposes: information management, large-scale deliberation, decision making, etc." [35]. Poblet et al. classified a set of 130 crowd-civic systems within different visions of citizenship, derived from liberal, republican, deliberative, and epistemic theories of democracy. Despite being limited in that they only focus on non-governmental initiatives, the classification provides an interesting approach to these sorts of service from a political perspective.

Another civic technology mapping initiative was conducted by the Civic Tech Field Guide [36], a crowdsourced, global collection of civic tech tools and projects, covering hundreds of platforms/services designed to support different dimensions of e-Participation: civic crowdfunding, participatory budgeting, open innovation, crowdlaw, or group decision-making, for instance. Most tools are not yet DLTs-based. This is the case, for example, of Consul [37], a free software citizen participation tool for open government projects at local level that is used by hundreds of institutions such as the municipalities of Madrid (Spain), Paris (France) and Buenos Aires (Argentina). Although Consul is scalable and has been fully tested in many large institutions around the world, its reliability, privacy and transparency cannot be fully guaranteed whereas DLTs-based solutions can. Another example is the nVotes Platform [38], which supports the complete voting process in institutions with a noteworthy focus on preserving cybersecurity and end-to-end verifiability thanks to its platform-based auditability management. As it is not a distributed system, its reliability and integrity depend on trust in this third party. 
This is an authors' version of the article published in "Neurocomputing". The final authenticated version is available online at: https://doi.org/10.1016/j.neucom.2020.03.116

The Civic Tech Field Guide has a section devoted to Blockchain initiatives with 37 results [36], including tools, reports, centers and projects. Among the tools identified, we would highlight Follow My Vote [39], a DLTs platform designed to guarantee electronic voting with high security and transparency. Its use does not compromise voter privacy, facilitating the traceability of their vote through the entire process until the final results.

Nevertheless, there is a gap in identifying and analyzing a DLTs solution for civic systems. To solve this the European Commission's Policy Lab has launched a project to map the European Ecosystem of Blockchains and DLTs for Social and Public Good [40].

In order to underline the innovative design of our proposed DLTs system, we have analyzed the following platforms which, to our knowledge, are currently the most significant:

- AGORA [41], a tool that uses Agora's VOTE token as a trading system in the secondary market. VOTE, as a native token, incentivizes citizens and selected organizations to contribute as authenticators to a secure and transparent election process worldwide. VOTE secures the vote through a payment system.

- Bitnation [42], a decentralized platform for e-Governance, deployed in 2014, that explores new approaches to democratic and social processes. To develop these social innovations, we use the Pangea project-a decentralized market for legal services, through which users can experience new citizen governance services. All their Smart Contracts are implemented in Ethereum.

- BoardRoom [43], a governance management system, integrating both a dashboard and a dApp that is used to manage Smart Contracts, in public as well as in permissioned blockchains developed in Ethereum. It supports the issue of tokens in crowdfunding projects, proxy voting systems for shareholders in committee or shareholders' meetings, and the development of payment systems through tokens in industrial consortia.

- Bobak [44], a "multijurisdictional" decentralized voting system, giving access to voters in different countries around the world. Like Voatz, Bobak is built on the The Hyperledger Project application set, specifically with Fabric. The Bobak platform is based on Ethereum's smart contract network.

- Coinstack [45], developed in 2016, works on the Bitcoin blockchain and is compatible with Ethereum smart contracts. This system was used by the South Korean province of Gyeonggi-do to vote on community projects.

- i-Voting [46], a system that allows voters to cast their ballots from any internet-connected computer anywhere in the world. Completely unrelated to the electronic voting systems used elsewhere, this application uses a GCloud framework deployed in Estonia. During a designated pre-voting period, the voter logs onto the system using an ID-card or Mobile-ID, and casts a ballot. The voter's identity is removed from the ballot before it reaches the National Electoral Commission for counting, thereby ensuring anonymity. As it was used in the most recent Estonian general elections, Estonia's solution was to allow voters to log on and vote as many times as they wanted to during the pre-voting period. Since each vote cancels the last, voters always have the option of changing their vote until the close of the elections.

- Polys [47], an Ethereum smart contract-based system, launched in November 2017 that applies a DLTs system to e-Voting. To ensure that each vote is anonymous, voting calculations are encrypted. This is a freemium model (some actions are paid) and is modifiable.

- Secure Vote [48], a Bitcoin blockchain-based voting system available for smartphones or network-connected voting machines. They use an algorithm called Blockchain Agnostic Scalability Layer as a scaling off-chain solution. Secure vote was tested with a stress test that consisted of a massive vote of 1.5 billion verified votes in the blockchain.

- TIVI [49] uses an encryption system to secure votes. Voter authentication systems have been developed in Argentina and Armenia in 2017 and their first inclusion in systems of choice with Blockchain technology was in 2016 for the US Republican Party's primary elections in Utah. Their actions are more oriented to the automatization of electoral processes than to the development of e-Participation systems.

- Voatz [50], a voting platform first deployed in March 2018 and accessible by invitation, which combines blockchain with biometric technology to authenticate voter identity. The West Virginia Senate used this tool so that prominent military personnel stationed abroad and other authorized Americans living outside the United States could vote. Voters only require a valid ID and a mobile device with Android or iOS operating systems.

Our proposal differs from the DLTs e-Voting systems previously described as follows:

a) Our system uses a virtual token to reward citizen participation; this can be used at discretion, following a given chart of rights and/or services proposed by the institution.

b) The virtual token is deployed through a smart contract that the block itself produces within the RETIS framework. This block contains the transaction (the vote) and all the documents related to the process (ID, census certificate, etc.).

c) There is no gas for any transaction in RETIS. 
This is an authors' version of the article published in "Neurocomputing". The final authenticated version is available online at: https://doi.org/10.1016/j.neucom.2020.03.116

d) The NDL EQUO framework facilitates the interconnection of blockchain networks in a transparent, certified, secure, auditable, scalable and traceable way. It can create virtual markets in order to offer unified products and services hosted by different Blockchain networks. They include several dApps used for e-Democracy purposes.

Before progressing to more technical issues, we would like to stress that, from the perspective of the literature on e-Participation, the inclusion of rights as a way of recognizing participation in a process is intended as a means of addressing a fundamental problem in direct democracy: the lack of the motivation to participate in certain cases [51]. As Naranjo-Zolotov et al. [30] stated, a decision-making-based participation model requires "an active engagement and involvement of the citizens in the long-term scenario for its success". Edelmann et al. [52] posed the question in other terms: "Given that lurkers make up a majority in the e-participation process, the crucial question is how we can motivate them to become active". This is the main challenge that we aim to resolve by providing tangible incentives to potential participants in participatory processes who would not simply participate because of their intrinsic civic motivation.

In cases in which privacy and security could be deterrents to participation, Blockchain-based systems have an added value for the voter given that a layer of cryptographic security anonymizes and guarantees both the nonviolability of the process and data protection. The use of a dApp as proposed in this study, also opens up the opportunity of developing participatory processes involving elements of gamification [53]. That is, the possibility of playing an active role in these processes with an emotional or practical counterpart, which is recognized by the local administration.

Because of this, the use of DLTs is a clear opportunity to develop new e-Participation models, with new tools and a new approach. These considerations are all included in the e-Participation Index study in order to integrate the governmental and social perspectives [54] in this kind of e-Democracy process.

\section{New approach to a "Tokenizable" e-Participation model}

\subsection{The Governance deficit}

Currently the concept of governance is devoid of meaning. The complexity of society, the liquid components of relationships, and economic and social uncertainty have led citizens to demand a new type of relationship with public administrations that is based on greater transparency and more participatory systems. All of which, in a digital context, generates a demand for new forms of e-Participation [10].

The current dominant paradigm is the result of a deliberate change that has placed citizens at the center of the new democratic governance model [55] that is the result of phenomena such as Open government [56], digital participation and e-Government [57]. The New Public Management paradigm [58] has been replaced by a newer paradigm that comes under names like "Networked Governance" [59], "Public Service Dominant" [60] or "citizen-centered governance" [61].

Therefore, we need to design and establish an e-Participation system that could regularly complement the representative model of governance in our democratic societies.

\subsection{The roles of Open Smart Cities and "smart citizens": opening local governments}

Our approach is part of the open government systems that applies a set of digital tools to develop e-Democracy solutions in the context of public participation. This obliges us to reconsider the relationship between technology, people and cities from the perspective of citizenship, with its complex and contradictory vision of the urban concept [62].

Smart City governance models [63] are possible thanks to technology-based solutions that take advantage of Big Data frameworks and lead to improved decision-making schemes. The danger of a deterministic model lies in building and trusting automatized technocratic decision-making systems based on data exploitation by artificial intelligence, rather than on consensual public policies formed by the active participation of empowered citizens. Therefore, in the same way that the Open Government concept [64] has been developed to establish new participation and accountability systems [65], we need to establish a new concept: the Open Smart City.

The Open Smart City is a philosophy that implies empowering citizens. It involves putting cities at the service of their citizens, empowering, in turn, public officials to develop policies that are defined and agreed upon by the parties 
This is an authors' version of the article published in "Neurocomputing". The final authenticated version is available online at: https://doi.org/10.1016/j.neucom.2020.03.116

involved. It is the paradigm of governability (the form of governing), which supports governance (the processes and protocols that facilitate governing), and therefore requires a more complex, permanent effort.

This vision implies giving space to new bottom-up dynamics that come from society and incorporate innovative collective intelligence into public management. Some examples are citizen science, digital activism, "artivism", the "maker" movement, the co-creation and self-development communities, digital citizen movements, social hackers, citizen innovation laboratories [66], among others. In some cases, government itself, at different levels, has opened up its bureaucratic innovation procedures to incorporate stakeholders' views, as in the creation of policy and government labs [67].

The idea we present is intended to "open" the data and the processes that cities generate, to make them available to society, converting them into common practice in the service of people. This provides an opportunity to create more democratic co-decision spaces in the political arena, creating the figure of Open Smart Citizens, i.e. citizens who operate in the spaces described above, and offers a new opportunity to generate new public policies and new democratic spaces. This approach allows active citizens to take part in deliberations over policies and the decisionmaking process. These kinds of citizen are identified because they: 1) use data produced by the city; 2) share their own data to generate a more intelligent system; and 3) participate, to different degrees of involvement at will, in the governance of the city through e-Participation models and platforms.

Data mining and big data facilitate the development of new tools and techniques to manage the city and its social diversity from the point of view of social innovation.

In fact, some of the concepts we discuss in the present article have not yet been adopted by the e-Government narrative. The key concepts of this article, such as "smart citizen", "government as a platform" and "open government," have not been fully incorporated into the 100 most frequently cited e-Government studies, as reflected in that coordinated by Dirk Draheim [68]. This means that we need to develop new DLTs-based e-Participation models that will facilitate the generation of new tools to improve our democratic systems in order to guarantee the engagement processes between actors in the political game.

Our proposal aims to support the growing demand of citizens to participate actively in the decisions that affect cities and the evolution of data mining techniques and their visualization tools. These allow for information to be available in real time, giving people the opportunity to have immediate data to manage personal responsibility in the creation and design of services that affect them directly [69]. These co-creation and co-participation processes are within the fundamental principles of open government and are what facilitates the deployment of those elements that make up the Open Smart Cities format.

\subsection{A "tokenizable" e-Participation model}

Our model (Fig.1) is based on a public G-Cloud system that allows a blockchain platform to be integrated as a service (BaaS). This guarantees the functional requirement of scalability necessary in any governmental and/or territorial environment. In our system, the voter would access the platform through a permissioned dApp that would connect with the interface and the voter database to proceed with the recognition and identification of a specific person in the process.

Then, the System Administrator would activate the Smart Contract at the front end that would allow the citizen to participate in the process opened by the agency to register their participation and their vote. In the validation node, data encryption is performed with a one-way SHA-256 hash function so that the result of voting cannot be reversed. Once the vote is encrypted, the block will be added to the blockchain within the BaaS architecture used and, in turn, the block generates the "token" (through a transaction generated by a smart contract as explained in section 4) which will grant the voter a series of advantages and/or rights of use in the city. 
This is an authors' version of the article published in "Neurocomputing". The final authenticated version is available online at: https://doi.org/10.1016/j.neucom.2020.03.116

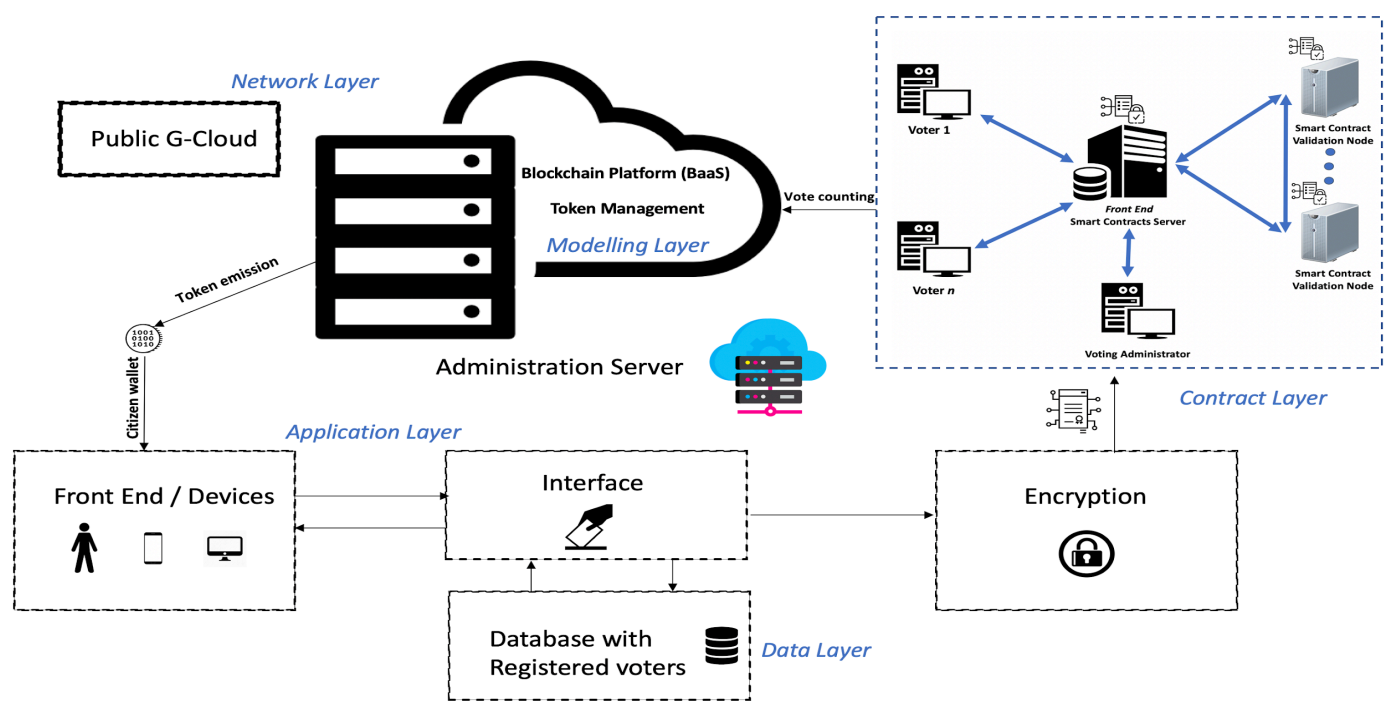

Fig. 1. Simplified representation of the proposed Blockchain e-Participation framework

To develop this proposal we have taken into account previous studies of electronic voting that are being conducted in parallel by blockchain to solve different technical issues for its progressive implementation. Specifically:

a. Those that differentiate the use of blockchain to develop voting systems using crypto currencies [70], such as those that use the Zcash protocol [71];

b. Those that rely on the deployment of Smart Contracts [72] with the limitations of options and number of voters that this entails, as is the case of BoardRoom; and,

c. Those that manage the blockchain as a "ballot box," like FollowMyVote and TIVI [73].

Some studies have developed electronic voting systems that establish taxonomies and requirements [74] [75], the conceptualization of how to use blockchain to develop voting systems [76], or how to develop voting systems that provide voters with more control of the process and its decisions [77].

Our proposal takes account of the large repository of projects and academic articles on the implementation of cryptocurrencies available in order to apply "tokens" in a practical way in e-Democracy environments and to overcome the concept of Fintech solutions in relation to token generation.

At present, Ethereum is the platform most commonly used to develop applications using smart contracts and tokens in a more efficient way. This public blockchain includes, amongst others, one very specific token: ERC721 [78]. This is defined as a non-expendable token that is unique and non-reproducible and is associated with a specific asset (or right). Initially, this type of token could be a solution for the electronic voting process, associating one vote (unique, with a specific choice) with a single citizen. But that is not our intention as authors of the present proposal. Our intention is to associate the token of the vote with a right to participate that could be exchanged for different types of goods or benefits, (for example, exemption from, or a reduction in fees or taxes, to obtain tickets for cultural or sports events, access to training activities, etc.). Given this orientation of the token, it makes no sense to establish a proper, unequivocal and non-transferable relationship with its value since, when the token is used (exchanged on the management platform of the municipal website), it will be eliminated. If the token disappears (since it is issued for a specific function, associated to a single smart contract) it makes no sense to use ERC721, the existence of which is determined by giving value to a property (or asset) that is transmissible and has an intrinsic value assigned.

Similarly, the operation of smart contracts in other public blockchain platforms such as Hyperledger Fabric and the like, does not help us design our proposal. Essentially, there are two reasons for this. The main one is because in RETIS we use the Smart Contract as a blockchain block that includes the transaction and all the document management and digital identity of the voting system, voter by voter. And secondly, it does not originate transaction prices. There is no gas for each transaction, nor does it need highly inefficient work tests.

Therefore, we understand that our system differs from those developed so far because both the management system of smart contracts (as blocks of the blockchain itself) and the non-generation of any type of economic exchange for the transaction (gas) is more efficient and scalable than those based on Ethereum. 
This is an authors' version of the article published in "Neurocomputing". The final authenticated version is available online at: https://doi.org/10.1016/j.neucom.2020.03.116

Our proposal aims to use these new "tokens" to reward participation and co-management of projects of public value. Tokens will be generated from each participant's vote and will be associated to a smart contract in the process which determines this [79], as stated in section 4.

These kinds of token can be used to obtain different benefits and can be accumulated or exchanged by individuals. Also, these tokens can be used as proof of civic values and be part of the citizen's civic or/and professional portfolio.

Our proposal is expected to raise the participation ratios in public participatory processes and votes, as well as in social innovation and co-creation projects in which citizens' contributions, particularly those of younger generations, may require providing visibility and recognition.

\section{$4 \quad$ A Neural Distributed Ledger (NDL): one step beyond}

\subsection{Introducing RETIS}

To develop a novel, more secure, scalable and reliable e-Participation public space, we propose a collaborative and multidimensional Blockchain network. This blockchain is internally structured analogously to the way neuron groups are structured in the human brain. Hence, we have termed it a Neural Distributed Ledger (NDL). The way in which our proposed ledger operates is by adding all the "memories" (transactions) stored in the clusters that make up the platform. The name we have given this type of network is RETIS (currently in the process of being patented).

The idea of establishing a neural system to develop a blockchain as "personal thinking chains" was defined by Swan [80] in 2015 as a bridge towards the integration of AI and IoT for new applications and types of architecture as universal transaction systems. RETIS is a first step towards a deeper understanding of the visionary concept outlined in the aforementioned article.

In one of the most recent studies on types of blockchain-based utilities and applications - particularly those related to e-Voting systems - no system has been identified as possessing the features of our proposal [81]. This platform is specifically designed to meet the requirements of business and government environments that need to consume and process massive data for their management processes. Our proposed platform, which is currently under development, is a private infrastructure network that hosts mixed public and private information in all its nodes.

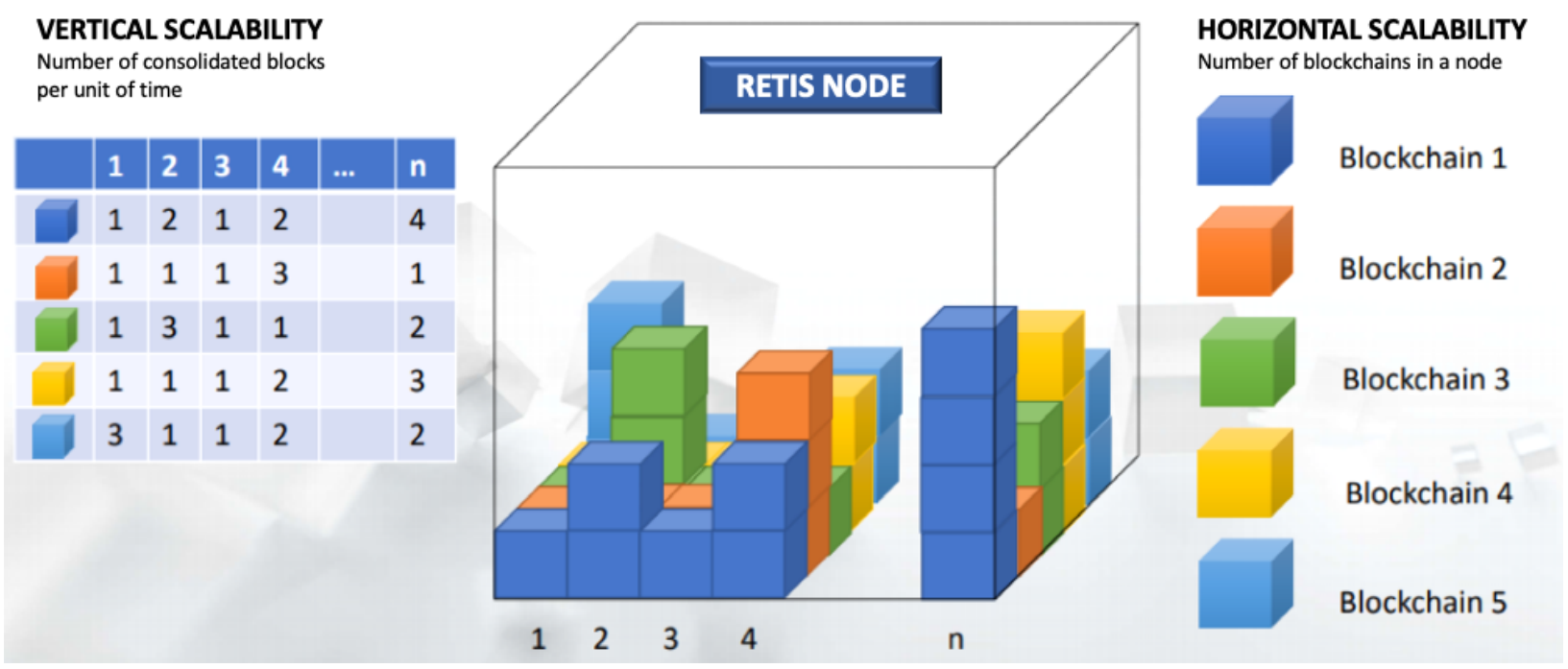

Fig. 2. Why RETIS is a 3D blockchain network

Figure 2 helps us understand why RETIS acts as a three-dimensional network which accumulates nodes in different blockchains within the platform, while building blocks in its different blockchains. This ensures vertical and horizontal scalability that forms a 3D mesh to deal with the massive data management and transactions that the platform must support per unit of time.

RETIS was designed in the expectation that there would be large numbers of movements (transactions) that must be processed by several nodes which, in turn, will collaborate to register as many of them as possible, in each turn. 
This is an authors' version of the article published in "Neurocomputing". The final authenticated version is available online at: https://doi.org/10.1016/j.neucom.2020.03.116

The use of this new operating paradigm invalidates the possibility of using traditional consensus algorithms such as POW, POS and the like, makes the network highly sustainable, and develops another typology of Byzantine fault tolerance. Therefore, the RETIS network implements specific algorithms for network governance. This requires the development of a new node management model to achieve a new type of governance adapted to the new validation requirements. This network is fully prepared for use in governance of the Sustainable Development Goals (SDGs), given its sustainability and its ability to scale the document transaction needs of any institution or organization. It can be particularly effective in accomplishing SDG 16: "[To] promote peaceful and inclusive societies for sustainable development, provide access to justice for all and build effective, accountable and inclusive institutions at all levels".

\subsubsection{How do RETIS nodes work?}

Each node begins by operating in a Passive mode, which means that it will wait until it receives a client connection through a valid access point that complies with the exposed services API, in order to read or modify the status of Active nodes. RETIS nodes display dual behavior: they are in Passive mode when they respond to customer requests and in Active mode when they generate movements based on programmed procedures. In some special cases the Active mode will be enabled by the node owner. When this occurs, the node periodically executes a set of scheduled tasks that allow it to act as an automatic broker within the network. These tasks are often used to execute automatic transactions, maintenance tasks or data clearing.

\subsubsection{RETIS and Smart Contracts}

Smart Contracts are the most controversial feature of Blockchain platforms, given that they each define and implement the Smart Contracts according to their own standard and definition. In fact, the cryptographer Szabo defined Smart Contract in the 1990s as "a set of promises, specified in digital form, including protocols within which the parties perform on these promises". [82]

If we take the Szabo operating framework into account, RETIS works with its own Sandbox Execution Environment (SEE) within the private blockchain that runs with an execution system similar to that used in Hyperledger Fabric [83].

RETIS has been designed to provide all the functionality that the main platforms on the market call Smart Contract, as node capability-embedded. This is especially the case in projects designed to manage IoT data or related to the execution of Machine Learning processes. This approach was previously defined as a DAO (Decentralised Autonomous Organization), but in this case this type of Smart Contract is deployed in a private network instead of a public one [84].

Table 1 shows different models of Smart Contracts and how RETIS uses them.

\begin{tabular}{|l|l|l|}
\hline Platform & How the Smart Contract is deployed & How does RETIS do this? \\
\hline ETHEREUM & $\begin{array}{l}\text { A script must be launched on receipt of a certain } \\
\text { type of transaction in the blockchain to execute } \\
\text { certain tasks. }\end{array}$ & $\begin{array}{l}\text { Using the behaviors and scheduled } \\
\text { tasks of the node (ACTIVE and } \\
\text { PASSIVE MODE). }\end{array}$ \\
\hline HYPERLEDGER & $\begin{array}{l}\text { An API of microservices that regulate read-or- } \\
\text { write access to the underlying blockchain. }\end{array}$ & $\begin{array}{l}\text { Using services published by node } \\
\text { access points (Entry Points). }\end{array}$ \\
\hline RETIS & $\begin{array}{l}\text { An administrative file defines the nature and } \\
\text { execution of a business process and its } \\
\text { traceability during its lifetime. }\end{array}$ & $\begin{array}{l}\text { Each Smart Contract corresponds to a } \\
\text { specific type of entity that meets the } \\
\text { Smart Contract template definitions. }\end{array}$ \\
\hline
\end{tabular}

Table 1. Comparative list in the deployment of smart contracts 
This is an authors' version of the article published in "Neurocomputing". The final authenticated version is available online at: https://doi.org/10.1016/j.neucom.2020.03.116

\subsubsection{How does a Smart Contract in RETIS work?}

Following the scheme in Figure 3, the RETIS network is used thus:

1. To identify participants and signers, using a decentralized Digital Identity architecture within the RETIS framework, using a CP/AP system (Certification Provider/Authentication Provider) in a BaaS context as an "end-user service provider" with human and/or machine users.

2. Building a workflow template to simplify and standardize the specifications of transactions that will be developed in RETIS by the network of users who access the services.

3. Adding legal and other required documents for secure document transfer and transactions based on these.

4. Publishing to the NDL Network for tamper-proof and secure storage in the private neuronal blockchain.

5. On Demand Execution, all the transactions are allowed and triggered by the blockchain nodes and users with permission to do so.

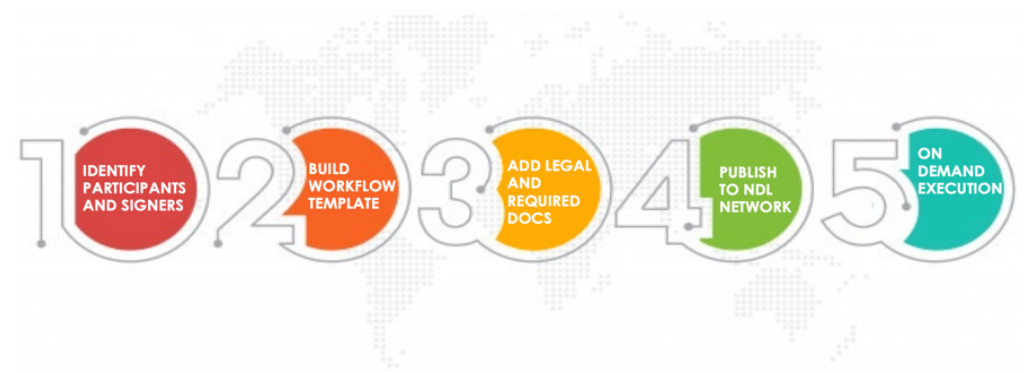

Fig. 3. RETIS Smart Contracts scheme

This blockchain is not designed to issue fixed tokens, since the system understands the token as a most valued object that is managed and stored in the blockchain. RETIS associates the token with an identification field. In RETIS, a token is generated by having a transaction that applies a change to an asset. This new asset, generates a new transaction that "moves" a token from one wallet (the user's dApp) to another. This means that our network does not have or generate "gas", which eliminates a calculation complexity that could slow down the network when it determines the number of transactions per block. From this point of view, we understand that it is an ideal environment for the management of e-Participation projects.

VoteKeeper is the dApp that materializes the vote in the RETIS Network. With this application we certify the vote and the transaction that produces it, and it is stored as a virtual "token" that issues the participatory right within it, to be used by the voter in the manner provided for by the platform. Figure 4 shows the conceptual framework of the network operation to register and validate the vote within the network. 
This is an authors' version of the article published in "Neurocomputing". The final authenticated version is available online at: https://doi.org/10.1016/j.neucom.2020.03.116

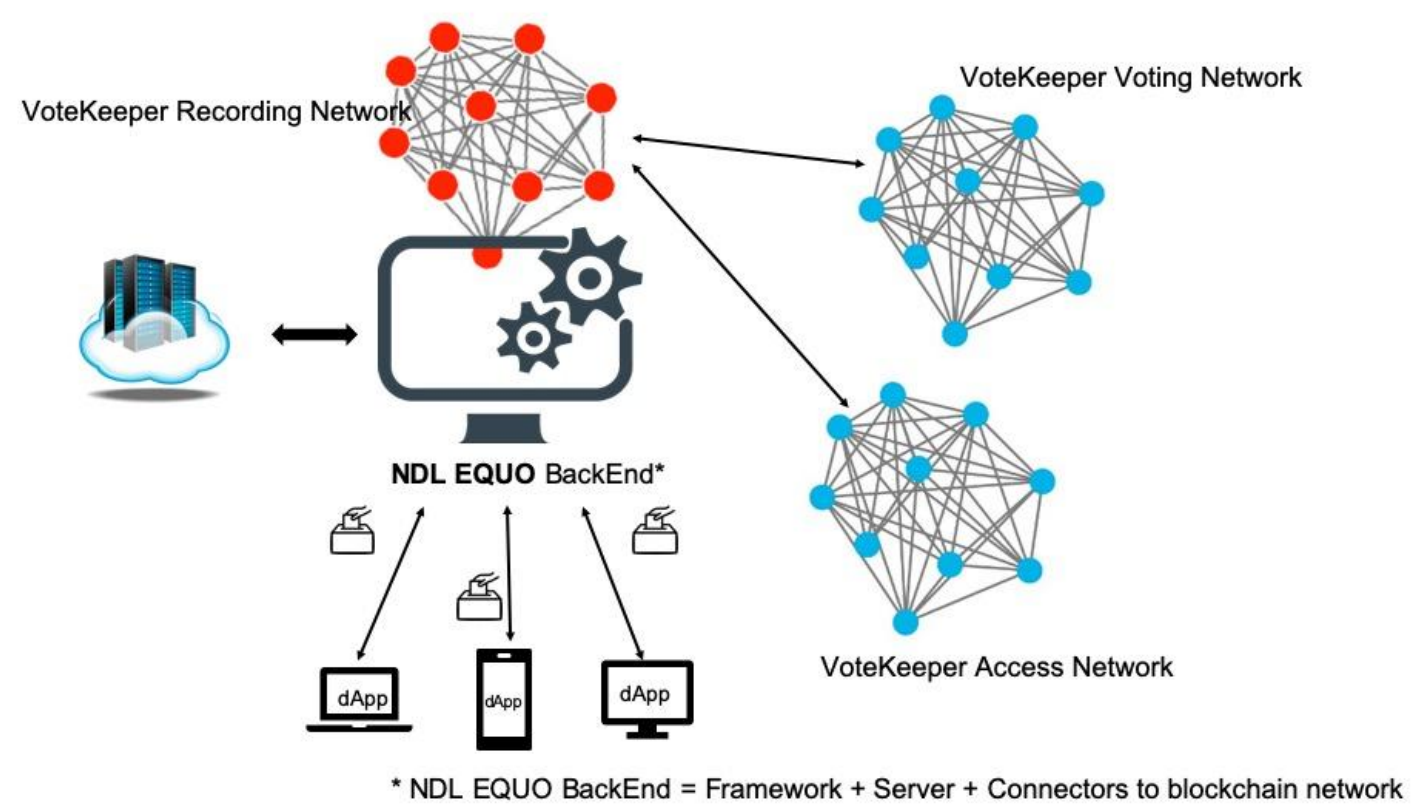

Fig. 4. VoteKeeper dApp conceptual framework

\section{The Second level blockchain called INTEGRA}

Within the proposed framework, INTEGRA is a second level Blockchain network (based on RETIS) the purpose of which is to interconnect other Blockchain networks to establish cross-cutting and scalable asset markets. The search for scalable governance and the definition of standards of use [85] [86] is one of the issues we intend to develop within our proposed model. This facilitates the integration of other existing blockchains, regardless of their type: public, private or consortium.

It operates by creating the appropriate framework to be able to define the following capacities of the proposed network:

1. To interconnect Blockchain networks in a transparent, certified, secure, auditable and traceable way.

2. To create virtual markets that offer unified products and services hosted on different Blockchain networks.

3. To define new products and services by combining those that offer interconnected networks

All RETIS network services and their management are based on a BaaS model and contained in a cloud model in order to permit rapid adaptability to the needs of any organization and, especially in government environments, to facilitate a rapid response. The system proposes a dApps-based work scheme available to any institutional network user.

\section{$5 \quad$ Conclusions and Future Work}

Currently we need to experiment with new methods that encourage the greater commitment of citizens to their local institutions. Blockchain technology can use tokens as a tool to increase civic engagement by supporting citizens' extrinsic motivations.

In the present study, we have proposed a blockchain-based model for the management of local governance as a more efficient and more inclusive approach that opens up new paths for the local management of territories. Its deployment follows a BaaS approach, which ensures scalability in a G-Cloud solution environment within a neural blockchain network. This enables the development of e-Democratic environments that facilitate better management and increased citizen engagement. 
This is an authors' version of the article published in "Neurocomputing". The final authenticated version is available online at: https://doi.org/10.1016/j.neucom.2020.03.116

Our proposal is based on the use of "Virtual Tokens" to increase and reward citizen participation and promote the co-management of public utility projects. It is aimed at finding new forms of commitment that can strengthen local democracies because technological deployment in the last decade has not been accompanied by greater participation or greater resilience of our democracies given the spaces of uncertainty generated by our complex societies.

In section 2, we have presented solutions that show the technological answers available to date and highlight the novelty of our proposal as a means of addressing the existing deficit in citizen governance.

Neural blockchain technology applied to governance processes has yet to be developed. Therefore, an opportunity exists to experiment with new tools and new e-Participation formats to help consolidate and improve our democratic systems in more participatory ways.

Our proposal presents a new conception of blockchain that facilitates the use of a virtual token to reward citizen participation. The virtual token is deployed through a smart contract that the block itself produces. This block contains the transaction (the vote) and all the documents related to the process (ID, census certificate, etc.) and there is, therefore, no gas for any transaction in the RETIS network. The front end is a dApp (VoteKeeper) than can be used in any electronic device (smart mobile, tablet or desktop computer). Finally, the NDL EQUO framework facilitates the interconnection of blockchain networks in a transparent, certified, secure, auditable, scalable and traceable way.

This model will be tested by the government of the province of Granada (Diputación de Granada). As part of the Integrated Sustainable Urban Development project, the University of Granada MediaLab is responsible for a participatory project in urban innovative action. Furthermore, we are currently working with the Spanish Smart City Cluster [87] in order to develop a pilot project to be deployed in several municipalities in Spain.

As future lines of research, the UTAUT model (Unified Theory of Acceptance and Use of Technology) [88] will be used to determine how this e-Participation model is received by citizens and which variables condition its adoption.

In both projects, "participatory tokens" are a strategic element of our expected results. These results will be indicators of the degree of success of the model presented in the present study, in the final quarter of 2020.

\section{Acknowledgements}

We would like to thank the ByEvolution [89] team for offering us the use of their proposed neural blockchain for e-Participation projects and the technological modifications that it implies, and 3CS Strategic Changes [90] for their collaboration in helping to develop the strategic framework that facilitates its deployment under the conditions provided in institutional environments. We especially want to thank Carlos Velasco, Computer Engineer and CTO of ByEvolution for his commitment to the development of this project.

\section{References}

1. S. Nakamoto, Bitcoin: A peer-to-peer electronic cash system. https://bitcoin.org/files/bitcoin-paper/bitcoin_es.pdf, 2008 (accessed 7 September 2019)

2. Yavuz, E., et al., Towards secure e-voting using Ethereum blockchain, in: 6th International Symposium on Digital Forensic and Security (ISDFS) (pp. 1-7). IEEE. (2018). doi: 10.1109/ISDFS.2018.8355340

3. HEDERA Hashgraph Platform, https://www.hedera.com/platform, 2017 (accessed 7 January 2020)

4. IOTA Foundation Tangle protocol,_https://www.iota.org/research/meet-the-tangle, 2017 (accessed 7 January 2020)

5. Cheng, S., et al., Using blockchain to improve data management in the public sector. McKinsey Digital. https://www.mckinsey.com/business-functions/mckinsey-digital/our-insights/using-blockchain-to-improve-datamanagement-in-the-public-sector, 2017 (accessed 19 January 2020)

6. C. Alexopoulos, Y. Charalabidis, A. Androutsopoulou, Benefits and Obstacles of Blockchain Applications in eGovernment, in: Proceedings of the 52 Hawaii International Conference on System Sciences, pp. 3377-3386. (2019). doi: 10.24251/HICSS.2019.408

7. Welch, E.W., The Rise of Participative Technologies in Government. Transformational Government Through eGov Practice: Socio-Economic, Cultural, and Technological Issues, Emerald Group Publishing Limited, Bingley, pp. 347-367. (2012)

8. Arnstein, S. R., A ladder of citizen participation. Journal of the American Institute of planners, 35(4), 216-224. (1969). doi: https://doi.org/10.1080/01944366908977225

9. Pateman, C., Participation and Democratic Theory. Cambridge University Press, London. (1970)

10. Macintosh, A., \& Whyte, A., Towards an evaluation framework for e-Participation. Transforming government: People, process and policy, 2(1), 16-30. (2008). doi: 10.1108/17506160810862928

11. K. Zhang, H. Jacobsen, Towards Dependable, Scalable, and Pervasive Distributed Ledgers with Blockchains, in: IEEE 38th International Conference on Distributed Computing Systems (2018), doi: 10.1109/ICDCS.2018.00134

12. Schmidthuber, L. and Hilgers, D. , "Unleashing innovation beyond organizational boundaries: exploring citizen sourcing projects", International Journal of Public Administration, Vol. 41 No. 4, pp. 268-283. (2018) 
This is an authors' version of the article published in "Neurocomputing". The final authenticated version is available online at: https://doi.org/10.1016/j.neucom.2020.03.116

13. J. Huang, A. Karduck, A Methodology for Digital Government Transformation, in: Journal of Economics, Business and Management, vol. 5, no. 6, pp. 246-254, (2017). doi: 10.18178/joebm.2017.5.6.521

14. F. Glaser, et al., Blockchain as a Platform, in: Business Transformation through Blockchain. Vol I. Treiblmaier, H. and Beck, R. (eds.). Palgrave MacMillan, pp. 121 - 143, (2019). https://doi.org/10.1007/978-3-319-98911-2 4

15. V. Morabito, Blockchain governance, in: Business innovation through Blockchain: The B Perspective, pp. 41-59. Springer International Publishing, (2017). doi: 10.1007/978-3-319-48478-5

16. F. Gräbe, et al., Do Not Be Fooled: Toward a Holistic Comparison of Distributed Ledger Technology Designs, in: 53rd Hawaii International Conference on System Sciences, (2020).

17. M. Rauchs, et al., Distributed Ledger Technology Systems. A conceptual framework, in: Cambridge Centre for Alternative Finance, (2018).

18. M. Alharby and A. V. Moorsel, Blockchain-based smart contracts: A systematic mapping study, in Proceedings International Conference in Artificial Intelligence Soft Computing, pp. 125-140, (2017). doi:10.1109/TSMC.2019.2895123

19. H. Watanabe, et al., Blockchain contract: A complete consensus using blockchain, in: Proceedings IEEE 4th Global Conference on Consumers Electronics (GCCE), pp. 577-578, (2015). doi: 10.1109/GCCE.2015.7398721

20. S. Gholami, The elimination of miners and block producers by using smart contracts, https://medium.com/swlh/theelimination-of-miners-and-block-producers-by-using-smart-contracts-58b20e317e9b, 2018 (accessed 22 October 2019)

21. S. Gholami, M. Kazeminia, Smart Blockchain is the new generation of Blockchain networks, 2019 https://medium.com/@info.solidity/smart-blockchain-is-the-new-generation-of-blockchain-networks-2dd95cc2aba0, (accessed 29 September 2019) Paper. http://search.iiit.ac.in/uploads/CloudComputingForEGovernance.pdf, 2010 (accessed 12 September 2019)

23. M. Svitek, P. Skobelev, S. Kozhevnikov, Smart City 5.0 as an Urban Ecosystem of Smart Services, in: Service Oriented, Holonic and Multi-agent Manufacturing Systems for Industry of the Future. SOHOMA 2019. Studies in Computational Intelligence, vol 853. Springer, Cham, (2019). https://doi.org/10.1007/978-3-030-27477-1 33

24. M.H. Onik, M.H. Miraz, Performance Analytical Comparison of Blockchain-as-a-Service (BaaS) Platforms, in: Emerging Technologies in Computing. iCETiC 2019. Lecture Notes of the Institute for Computer Sciences, Social Informatics and Telecommunications Engineering, vol 285. Springer, Cham, (2019). doi: https://doi.org/10.1007/978-3-030-23943-5_1

25. Sæbø, Ø., Rose, J. and Skiftenes Flak, L., The shape of e-Participation: characterizing an emerging research area. In: Government Information Quarterly, Vol. 25 No. 3, pp. 400-428. (2008). doi: 10.1016/j.giq.2007.04.007

26. Van Dijk, J., Models of democracy and concepts of communication. In: Digital democracy: Issues of theory and practice, 30-53. (2000)

27. Saward, M. (Ed.), Democratic innovation: deliberation, representation and association. Routledge. (2003)

28. UNDESA Report 2013, Guidelines on Open Government Data for Citizen Engagement, a Report Published by Department of Economic and Social Affairs, the Division for Public Administration and Development Management, http://workspace.unpan.org/sites/Internet/Documents/Guidenlines\%20on\%20OGDCE\%20May17\%202013.pdf, (accessed 19 January 2020)

29. United Nations, E-government Survey 2014 E-Government for The Future we Want, New York, NY. https://www.un.org/en/development/desa/publications/e-government-survey-2014.html (accessed 20 January 2020)

30. Naranjo-Zolotov, M., Oliveira, T., \& Casteleyn, S., Citizens' intention to use and recommend e-participation: Drawing upon UTAUT and citizen empowerment. In: Information Technology \& People, 32(2), 364-386. (2019). doi: https://doi.org/10.1108/ITP-08-2017-0257

31. Kang, M., Understanding public engagement: conceptualizing and measuring its influence on supportive behavioral intentions. In: Journal of Public Relations Research, Vol. 26 No. 5, pp. 399-416. (2014). doi: https://doi.org/10.1080/1062726X.2014.956107

32. Omar, A., Weerakkody, V. and Sivarajah, U., Developing criteria for evaluating a multi-channel digitally enabled participatory budgeting platform. In: International Conference on Electronic Participation. Springer, pp. 3-11. (2017). doi: 10.1007/978-3-319-64322-9_1

33. A. Macintosh, E-democracy and e-participation research in Europe, in: Digital government, Springer, Boston, MA, pp. 85102, (2008). https://doi.org/10.1007/978-0-387-71611-4_5

34. McInnis B., et al., Crowdsourcing law and policy: a design-thinking approach to crowd-civic systems. In: Companion of the 2017 ACM conference on computer supported cooperative work and social computing, 355-361. (2017). doi: https://doi.org/10.1145/3022198.3022656

35. Poblet, M., Casanovas, P., \& Rodríguez-Doncel, V., Linked Democracy: Foundations, tools, and applications. Springer. (2019). doi: https://doi.org/10.1007/978-3-030-13363-4

36. Civic Tech Field Guide. https://civictech.guide/ (accessed 20 January 2020)

37. CONSUL Project: http://consulproject.org/en/index.html, (accessed 9 January 2020)

38. nVotes Platform. www.nvotes.com (accessed 19 January 2020)

39. Follow My Vote Platform: https://followmyvote.com/, (accessed 9 January 2020)

40. European Ecosystem of Blockchains and DLTs for Social and Public Good. https://blogs.ec.europa.eu/eupolicylab/dlt4good-mapping-the-european-ecosystem-of-blockchains-and-dlts-for-socialand-public-good/, (accessed 20 January 2020)

41. Agora's VOTE Token. www.agora.vote, (accessed 20 January 2020) 
This is an authors' version of the article published in "Neurocomputing". The final authenticated version is available online at: https://doi.org/10.1016/j.neucom.2020.03.116

42. Bitnation Governance 2.0 Platform: https://tse.bitnation.co/, (accessed 9 January 2020)

43. BoardRoom Platform: http://boardroom.to/\#About, (accessed 9 January 2020)

44. Bobak Platform. http://salvaria.com/smartLegalTech/blog/index.html, (accessed 20 January 2020)

45. Coinstack e-Voting solution. https://en.blocko.io/usecases/public/ (Accessed 20 January 2020)

46. i-Voting Estonian Platform. https://e-estonia.com/solutions/e-governance/i-voting/ (accessed 20 january 2020)

47. Polys e-Voting system. www.polys.me (accessed 20 January 2020)

48. Secure Vote Bitcoin voting system. www.secure.vote/ (accessed 20 January 2020)

49. TIVI Online Voting System: https://tivi.io/, (accessed 9 January 2020)

50. Voatz voting platform. www.voatz.com (accessed 20 January 2020)

51. Lyu, H. S., The public's e-participation capacity and motivation in Korea: A web survey analysis from a new institutionalist perspective. In: Journal of Information Technology \& Politics, 4(4), 65-79. (2008). doi: https://doi.org/10.1080/19331680801975789

52. Edelmann, N., Parycek, P., \& Schossbock, J., The unibrennt movement: a successful case of mobilising lurkers in a public sphere. International Journal of Electronic Governance, 4(1-2), 43-68. (2011). doi: 10.1504/IJEG.2011.041707

53. Sgueo, G., Games, Powers \& Democracies: Chapter 1. In: Games, Powers \& Democracies. Bocconi University Press. (2018). Available at SSRN: https://ssrn.com/abstract=3198753 (accessed 20 January 2020)

54. A. Pirannejad, M. Janssen, J. Rezaei, Towards a balanced E-Participation Index: Integrating government and society perspectives, in: Government Information Quarterly, (2019). https://doi.org/10.1016/j.giq.2019.101404

55. J. S. Dryzek, Deliberative Democracy and Beyond: Liberals, Critics, Contestations, in: https://www.oxfordscholarship.com/view/10.1093/019925043X.001.0001/acprof-9780199250431, last accessed 2019/10/12.

56. D. Lathrop, L. Ruma, Open Government: Collaboration, Transparency, and participation in Practice. O'Reilly Media, Sebastopol. (2010).

57. P. Dunleavy, et al., Digital Era Governance: IT Corporations, The State, and e-Government. Oxford University Press, New York. (2006).

58. T. Nam, Citizens' attitudes towards Open Government and Government 2.0, in: International Review of Administrative Sciences, 78(2), 346-368, (2012). https://doi.org/10.1177/0020852312438783

59. Bennington, J., \& Hartley, J., Pilots, paradigms and paradoxes: Changes in public sector governance and management in the UK. International Research Symposium on Public Sector Management, Barcelona, April. (2001)

60. Osborne, S. P., Radnor, Z., \& Nasi, G., A new theory for public service management? Toward a (public) service-dominant approach. American Review of Public Administration, 43(2), 135-158, (2013). https://doi.org/10.1177/0275074012466935

61. Hartley, J., Innovation in governance and public services: Past and present. Public Money and Management, 25, 27-34, (2005). DOI: $10.1111 /$ j.1467-9302.2005.00447.x

62. M. Fernández, Descifrar las Smart Cities: Qué queremos decir cuando hablamos de Smart Cities, pp. 183-185. Caligrama Editorial, Madrid (2016)

63. Ruhlandt, R.W.S., The governance of Smart Cities: A systematic literature review, in: Cities, 81 (2018) 1-23. https://doi.org/10.1016/j.cities.2018.02.014

64. Wirtz, B.W. and Birkmeyer, S., Open government: origin, development, and conceptual perspectives, in: International Journal of Public Administration, Vol. 38 No. 5, pp. 381-396, (2015). https://doi.org/10.1080/01900692.2014.942735

65. Lorenço, R.P., An analysis of open government portals: A perspective of transparency for accountability, in: Government Information Quarterly, Volume 32, Issue 3, pp. 323-332, (2015). https://doi.org/10.1016/j.giq.2015.05.006

66. LabIn Granada. Social Innovation Lab. https://labingranada.org/, last accessed 2019/10/11.

67. E. Romero-Frías, W. Arroyo-Machado, Policy Labs in Europe: Political Innovation, Structure and Content Analysis on Twitter, in: El profesional de la información, 27, (6), pp. 1181-1192 (2018). https://doi.org/10.3145/epi.2018.nov.02

68. D. Draheim, et al., On the Narratives and Background Narratives of e-Government, in: Hawaii International Conference on System Sciences 2020 (HICSS-53), (2020). doi: 10.13140/RG.2.2.28264.21768

69. M. Garriga-Portolá, J. López Ventura, The role of Open Government in Smart Cities, in: Open Government Public Administration and Information Technology 4. Springer Science + Business Media, New York, (2014). doi: 10.1007/9781-4614-9563-5 13

70. Z. Zhao, T.H.H. Chan, How to vote privately using bitcoin, in: International Conference on Information and Communications Security. Pp. 82-96. Springer, (2015). https://doi.org/10.1007/978-3-319-29814-6_8

71. D. Hopwood, et al., Zcash protocol specification. Tech. rep., 2016-1.10. Zerocoin Electric Coin Company, (2016).

72. P. McCorry, S.F. Shahandashti, F. Hao, A smart contract for boardroom voting with maximum voter privacy. IACR Cryptology ePrint Archive 2017, 110, (2017). doi:10.1007/978-3-319-70972-7_20

73. B. Yu, et al., Platform-independent Secure Blockchain-Based Voting System, in: Chen L., Manulis M., Schneider S. (eds) Information Security. ISC. Lecture Notes in Computer Science, vol 11060. Springer, Cham, pp. 369-386, (2018). doi:10.1007/978-3-319-99136-8 20

74. R. Annae, R. Freeland, and G. Theodoropoulos, E-voting requirements and implementation, in: The 9" IEEE CEC/EEE 2007. IEEE, 2007, pp. 382-392, (2007). doi: 10.1109/CEC-EEE.2007.42

75. K.H. Wang, et al., A review of contemporary e-voting: Requirements, technology, systems and usability, in: Data Science and Pattern Recognition, vol. 1, no. 1, pp - 31-47, (2017). 
This is an authors' version of the article published in "Neurocomputing". The final authenticated version is available online at: https://doi.org/10.1016/j.neucom.2020.03.116

76. A.B. Ayed, A conceptual secure blockchain-based electronic voting system, in: International Journal of Network Security \& Its Applications, vol. 9, no. 3, (2017).

77. F.S. Hardwick, A. Gioulis, R.N. Akram, E-Voting with Blockchain: An e-Voting Protocol with Decentralisation and Voter Privacy, in: ISG-SCC, Royal Holloway, University of London, UK, (2018). DOI: 10.1109/Cybermatics_2018.2018.00262

78. ERC-721, www.erc721.org (accessed 29 January 2020)

79. F. Hjálmarsson, et al., Blockchain-Based E-Voting System, in: 2018 IEEE 11th International Conference on Cloud Computing, (2018). doi: 10.1109/CLOUD.2018.00151

80. M. Swan, Blockchain Thinking: The Brain as a Decentralized Autonomous Corporation, in: IEEE Technology and Society Magazine, December 2015, pp. 41-52, (2015). doi: 10.1109/MTS.2015.2494358

81. S. Aggarwal, et al., Blockchain for smart communities: Applications, challenges and opportunities, in: Journal of Network and Computer Applications, (2019). https://doi.org/10.1016/j.jnca.2019.06.018

82. N. Szabo, Smart Contracts: Building Blocks for Digital Markets. http://www.fon.hum.uva.nl/rob/Courses/InformationInSpeech/CDROM/Literature/LOTwinterschool2006/szabo.best.vw h.net/smart_contracts_2.html, 1996 (accessed 14 October 2019)

83. S. Wang, Y. Ouyang, Y. Yuan, Blockchain-Enabled Smart Contracts: Architecture, Applications, and Future Trends, in: IEEE Transactions on Systems, Man, and Cybernetics: Systems , pp. 1-12, (2019). doi: 10.1109/TSMC.2019.2895123

84. V. Shermin, Disrupting governance with blockchains and smart contracts, in: Special Issue: The Future of Money and Further Applications of the Blockchain, Volume 26, Issue 5, pp. 499-509, (2017). doi: 10.1002/jsc.2150

85. G. Benedict, Challenges of DLT-enabled Scalable Governance and the Role of Standards, in: Journal of ICT, Vol. 73 , 195-208. River Publishers, (2019). doi: 10.13052/jicts2245-800X.731

86. C. Aristidou, E. Markou, Blockchain Standards and Government Applications, in: Journal of ICT, Vol. 7 3, 287-312. River Publishers, (2019). doi: 10.13052/jicts2245-800X.736

87. Smart City Cluster, https://smartcitycluster.org/en/ (accessed 20 January 2020)

88. M. Naranjo-Zolotov, et al., Examining social capital and individual motivators to explain the adoption of online participation, in: Future Generation Computer Systems 92, pp. 302-311. Elsevier B.V., (2018). https://doi.org/10.1016/j.future.2018.09.044

89. ByEvolution Creative Factory, https://byevolution.com/?noredirect=en_US (accessed 20 January 2020)

90. 3CS Cambios Estratégicos, https://3cs.es/blockchain/ (accessed 20 January 2020) 